\title{
Transatlantica
}

Revue d'études américaines. American Studies Journal

James L.W. West III, ed. The Cambridge Edition of the Works of F. Scott Fitzgerald : "Trimalchio » : an Early Version of The Great Gatsby.

Cambridge : Cambridge UP, 2000. xxii-192 p. Illus.

Jean-Loup Bourget

(2) OpenEdition

Édition électronique

URL : http://journals.openedition.org/transatlantica/688

DOI : $10.4000 /$ transatlantica.688

ISSN : $1765-2766$

Éditeur

AFEA

Référence électronique

Jean-Loup Bourget, « James L.W. West III, ed. The Cambridge Edition of the Works of F. Scott Fitzgerald: "Trimalchio »: an Early Version of The Great Gatsby. », Transatlantica [En ligne], 1 | 2003, mis en ligne le 05 avril 2006, consulté le 29 avril 2021. URL : http://journals.openedition.org/transatlantica/688 ; DOI : https://doi.org/10.4000/transatlantica.688

Ce document a été généré automatiquement le 29 avril 2021

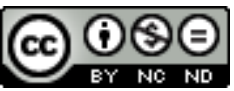

Transatlantica - Revue d'études américaines est mis à disposition selon les termes de la licence Creative Commons Attribution - Pas d'Utilisation Commerciale - Pas de Modification 4.0 International. 


\section{James L.W. West III, ed. The Cambridge Edition of the Works of $F$. Scott Fitzgerald : "Trimalchio »: an Early Version of The Great Gatsby.}

Cambridge : Cambridge UP, 2000. xxii-192 p. Illus.

Jean-Loup Bourget

1 Présenté comme " an early version of The Great Gatsby ", "Trimalchio" est une tentative de reconstitution du manuscrit dactylographié envoyé par Fitzgerald à son éditeur, Scribner's, en octobre 1924. Ce tapuscrit étant perdu, le texte a été établi à partir des épreuves avant correction, elles-mêmes composées à partir du tapuscrit. Il représente donc un stade intermédiaire entre le manuscrit holographe conservé à Princeton (facsimilé publié par les soins de Matthew J. Bruccoli en 1973) et le texte publié en avril 1925 qui nous est aujourd'hui familier. La publication de «Trimalchio» se justifie doublement.

D'abord, s'il était brouillé avec l'orthographe, Fitzgerald était aussi un styliste perfectionniste, ratureur obsessionnel, éternel insatisfait, qu'il est évidemment passionnant d'observer au travail. En outre, il tint certainement compte des remarques de Maxwell Perkins, son editor chez Scribner's, qui, enthousiasmé par le roman, avait néanmoins noté que le personnage de Gatsby n'était pas décrit avec suffisamment de netteté et que les révélations sur son passé et l'origine de sa fortune, venant tout à la fin du récit, auraient gagné à être préparées par quelques indices et à être étoffées sans que Gatsby y perde sa nécessaire aura de mystère. Pendant les quelques semaines qui séparent « Trimalchio » de Gatsby, Fizgerald procède donc à quantité de suppressions, d'ajouts, de modulations et d'étoffements, mais aussi à des changements de structure. Ajoutés, le passage décrivant le sourire de Gatsby, l'échange entre Gatsby et Nick sur l'impossibilité de faire revivre le passé. Etoffée, la confrontation entre Gatsby et Tom Buchanan à l'hôtel Plaza (dans « Trimalchio ", Daisy n'oppose guère de résistance à son 
mari, ce qui entraîne une défaite trop rapide de Gatsby). Surtout, les éléments qui éclairent le passé de Gatsby ont été déplacés pour figurer plus tôt dans le récit. Les réminiscences sentimentales de Gatsby passent du chapitre VII à la fin du chapitre VI, c'est-à-dire avant que le héros cesse de donner ses extravagantes réceptions et remplace son personnel stylé par des protégés de Wolfshiem. La relation des rapports entre Gatsby et son père adoptif Dan Cody passe du chapitre VIII (après l'accident dont est victime Myrtle Wilson) au début du chapitre VI; cette relation est en partie à la première personne dans "Trimalchio", alors que dans le texte publié Fitzgerald revient exclusivement à la troisième personne. Le rôle de Nick comme narrateur est donc renforcé au détriment de la voix propre de Gatsby, tandis qu'inversement sa responsabilité dans les retrouvailles de Gatsby avec Daisy s'estompe peu à peu du manuscrit à "Trimalchio», puis au texte publié.

Les réécritures et repentirs de Fitzgerald ne suivent pas toujours un schéma linéaire. En témoignent ses hésitations répétées sur le titre du roman: "The Great Gatsby", "Gatsby ", "Gold-hatted Gatsby », " The High-bouncing Lover », " Trimalchio in West Egg », « Trimalchio », « On the Road to West Egg », « Under the Red, White and Blue ». Il semble que le tapuscrit d'octobre 1924 ait été intitulé, comme le manuscrit holographe, "The Great Gatsby ", mais que Fitzgerald ait ensuite écrit à Perkins pour lui dire sa préférence pour "Trimalchio in West Egg ». Dépourvues de page de titre, les épreuves portent le titre courant « Fitzgerald's Trimalchio ». Seul Perkins aimait l'incongruité du rapprochement Trimalchio / West Egg; les autres (dont Zelda) jugeaient le titre trop cryptique, trop peu «vendeur». Fitzgerald continua à hésiter, préférant peut-être "Trimalchio » tout court (« imprononçable», selon Ring Lardner), mais il était trop tard : le livre était annoncé sous ce qui était, après tout, son titre original. Le parvenu Trimalcion n'avait pas réussi à détrôner Gatsby le Magnifique.

INDEX

Thèmes : Recensions

\section{AUTEUR}

JEAN-LOUP BOURGET

ENS Ulm 